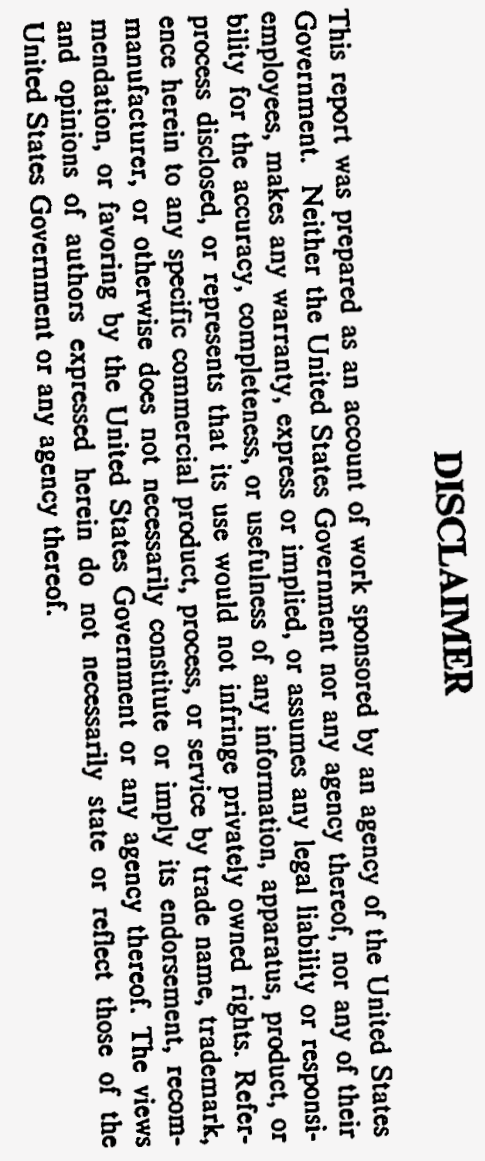

\title{
Global DC Closed Orbit Correction Experiment on the NSLS X-ray Ring
}

\author{
Y. Chung, G. Decker, and K. Evans
}

Advanced Photon Source

Argonne National Laboratory

Argonne, II 60439

In this note are described the global DC closed orbit correction experiments conducted on the X-ray ring at National Synchrotron Light Source (NSLS). The beam response matrix, defined as beam motion at BPM locations per unit kick by corrector magnets, was measured and then inverted using the technique of singular value decomposition (SVD). The product of the inverted matrix and the difference orbit gives the incremental kick strengths necessary to correct the orbit. As a result, the r.m.s. orbit error around the ring was reduced from $208 \mu \mathrm{m}$ to $61 \mu \mathrm{m}$. 


\section{DISCLAIMER}

Portions of this document may be illegible in electronic image products. Images are produced from the best available original document. 


\section{Introduction}

The third generation synchrotron light sources, such as the APS, are characterized by low emittance of the charged particle beams and high brightness of the photon beams radiated from insertion devices. Transverse stability of the particle beams is a crucial element in achieving these goals and the APS will implement extensive beam position feedback systems, which include 320 corrector magnets, 360 positron beam position monitors distributed around the storage ring, miniature BPMs for insertion device beam lines, and photon beam position monitors at the end of the $\mathrm{X}$-ray beam lines.

The beam position feedback systems can largely be divided into the global and local feedback systems according to the extent of correction, and the $\mathrm{DC}$ and $\mathrm{AC}$ feedback systems according to the bandwidth of correction. DC correction of the beam positions, as the name implies, is a slow process with sub- $\mathrm{Hz}$ bandwidth and is typically done with an integral control algorithm with unity gain for full correction and less-than-unity gain for partial correction. In contrast, AC correction is a fast process with wide bandwidth (typically $10-100 \mathrm{~Hz}$ ), and the APS will employ the proportional, integral, and derivative (PID) control algorithm to ensure stability with minimal noise infiltration. 1,2

In this note, we will present the results of global DC beam position feedback experiments conducted on the X-ray ring of the National Synchrotron Light Source (NSLS). Integral control with full correction was used, and the technique of singular value decomposition (SVD) was used to invert the response matrix. The product of the inverted matrix and the difference orbit gives the incremental kick strengths necessary to correct the orbit.

The rest of this note will consist of description of the theory of SVD for application to global beam position correction in Section 2 and the measurement results in Section 3. Summary will be given in Section 4 .

\section{Theory}

Global correction of the closed orbit is done with a set of corrector magnets distributed around the ring and a set of beam position monitors (BPMs). Let $M$ be the number of BPMs and let $\mathrm{N}$ be the number of correctors. Changes in the corrector strengths $\Delta \theta$ bring about changes in the closed orbit $\Delta \mathbf{x}$, and we assume that they are linearly related through the response matrix $R_{i j}$ by 


$$
\Delta x_{i}=\sum_{j=1}^{N} R_{i j} \Delta \theta_{j} . \quad(\text { for } 1 \leq \mathrm{i} \leq M)
$$

$\Delta x_{i}$ is the beam motion at the $i-$ th BPM and $\Delta \theta_{j}$ is the increment in the angular kick by the $j$ th corrector. The response matrix $R_{i j}$ can be written in terms of the betatron functions $\beta$ and $\psi$ at the locations of the BPMs and correctors as

$$
R_{i j}=\frac{\sqrt{\beta_{i} \beta_{c j}}}{2 \sin \pi \nu} \cos \left(\left|\psi_{i}-\psi_{c j}\right|-\pi \nu\right) \text {, }
$$

where $\beta_{i}$ and $\psi_{i}\left(\beta_{c j}\right.$ and $\left.\psi_{c j}\right)$ are the betatron amplitude and phase functions of the $i$-th BPM ( $j$-th corrector). $v$ is the tune.

The response matrix $R_{i j}$ in Eq. (2.1) can be directly measured by changing the strength of the $j$-th magnet by a small amount and then measuring the resulting beam motion at all BPMs and repeating the same procedure for all correctors. Global correction of the closed orbit is then equivalent to inverting this process. Writing Eq. (2.1) in matrix form, we have

$$
\Delta \mathbf{x}=\mathbf{R} \cdot \Delta \boldsymbol{\theta}
$$

The inverse matrix of $\mathbf{R}$, which we call $\mathbf{R}_{\mathrm{inv}}$, uniquely exists such that

$$
\mathbf{R} \cdot \mathbf{R}_{\mathrm{inv}}=\mathbf{R}_{\mathbf{i n v}} \cdot \mathbf{R}=\mathbf{1}
$$

if $\mathrm{M}=\mathrm{N}$ and if the matrix is not singular. 1 is the identity matrix.

Even if $M \neq N$ or if the matrix is singular, an inverse of the matrix can still be obtained, though with some restrictions, using the technique of singular value decomposition (SVD). ${ }^{3-5}$ Any $\mathrm{M} \times \mathrm{N}$ matrix $\mathrm{R}$ can be written as 6

$$
\mathbf{R}=\mathbf{U} \cdot \mathbf{W} \cdot \mathbf{V}^{\mathrm{T}} \text {, }
$$

where $\mathbf{U}$ is an $\mathbf{M} \times \mathbf{M}$ unitary matrix $\left(\mathbf{U}^{\mathrm{T}} \cdot \mathbf{U}=\mathbf{U} \cdot \mathbf{U}^{\mathbf{T}}=\mathbf{1}\right), \mathbf{W}$ is an $\mathbf{M} \times \mathbf{N}$ diagonal matrix with positive or zero elements, and $\mathrm{V}$ is an $\mathrm{N} \times \mathrm{N}$ unitary matrix $\left(\mathrm{V}^{\mathrm{T}} \cdot \mathrm{V}=\mathrm{V} \cdot \mathrm{V}^{\mathrm{T}}=\mathbf{1}\right)$. The representation given in Eq. (2.5) is unique only to a certain extent, and there are other ways of decomposing the matrix $R .7,8$

Since both $\mathbf{U}$ and $\mathbf{V}$ are unitary, they represent orthonormal transformations from one frame to another. The $\mathbf{V}$ matrix rotates the $\mathrm{N}$-dimensional orthogonal coordinate system, with each axis corresponding to a corrector magnet, to another $\mathrm{N}$-dimensional 
orthogonal coordinate system and generates a new set of $\mathrm{N}$ transformed correctors (or tcorrectors). The U matrix operates similarly on an M-dimensional BPM space and generates a new set of M transformed BPMs (or t-BPMs). Let us define $\Delta \mathrm{x}^{\mathrm{t}}$ and $\Delta \boldsymbol{\theta}^{\mathrm{t}}$ as

$$
\Delta x^{\mathrm{t}}=\mathrm{U}^{\mathrm{T}} \cdot \Delta \mathrm{x} \text { and } \quad \Delta \theta^{\mathrm{t}}=\mathrm{V}^{\mathrm{T}} \cdot \Delta \boldsymbol{\theta},
$$

where " $t$ " denotes "transformed". Then from Eqs. (2.3), (2.5), and (2.6), we have

$$
\Delta \mathbf{x}^{\mathrm{t}}=\mathbf{W} \cdot \Delta \boldsymbol{\theta}^{\mathrm{t}}
$$

Comparing Eqs. (2.3) and (2.7), we see that SVD diagonalized the matrix $\mathbf{R}$ into $W$. We can write the matrix $W$, with the indices $i$ and $j$ in the ranges $1 \leq i \leq M$ and $1 \leq j \leq$ $\mathrm{N}$, as

$$
W_{i j}= \begin{cases}w_{i} \delta_{i j}, & (M \leq N) \\ w_{j} \delta_{i j}, & (M \geq N)\end{cases}
$$

where $\delta_{i j}$ is the Kronecker delta. The diagonal elements $w_{i}$ 's (or $w_{j}$ 's), or the eigenvalues, are non-negative, and the number of them are equal to the lesser of $M$ and $N$. Associated with the eigenvalues are the mutually orthogonal eigenvectors $\left\{v_{j} \mid 1 \leq j \leq N\right\}$ spanning the space of the t-correctors. Similarly, we have $\left\{u_{i} \mid 1 \leq i \leq M\right)$, a set of mutually orthogonal unit vectors spanning the $t-B P M$ space. These eigenvectors are related by

$$
\mathbf{R} \cdot \mathbf{v}_{\mathbf{i}}=\mathrm{w}_{\mathbf{i}} \mathbf{u}_{\mathbf{i}}, \quad 1 \leq \mathrm{i} \leq \min (\mathrm{M}, \mathrm{N}) .
$$

Thus, the eigenvalues represent the coupling efficiency between the t-correctors and $t-$ BPMs.

Since the $W$ matrix has dimension $M \times N$, some of the columns are all zeroes when $M<N$ and some of the rows are all zeroes when $M>N$. Let us first consider the case when $M<N$. We can see immediately that there are at least $(N-M)$-correctors which have no corresponding non-zero eigenvalues and contribute nothing as far as orbit correction is concerned. Therefore, these t-correctors can be set to any arbitrary values, but for the purpose of minimizing the norm of the vector $\Delta \theta$, they are set to zero. Simiarly, if $M>N$, then we have at least $(M-N) t-B P M s$ which cannot be changed since they have no coupling to the correctors. This imposes a limitation on how much the closed orbit can be corrected with a given number of correctors. The finite capacity of the power supplies is another limiting factor and will be discussed later. 
One great advantage of SVD is that we can know in advance whether a given matrix is singular or not before trying to invert the matrix and remove the singularities if so desired. From Eq. (2.5), we write the inverse of the matrix $\mathbf{R}$ as

$$
\mathbf{R}_{\text {inv }}=\mathbf{V} \cdot \mathbf{W}_{\text {inv }} \cdot \mathbf{U}^{\mathrm{T}},
$$

where the $\mathrm{N} \times \mathrm{M}$ matrix $\mathbf{W}_{\text {inv }}$ is constructed by inverting the eigenvalues and then taking the transpose of the matrix. If $M \leq N, W \cdot W_{\text {inv }}$ is equal to the the $M \times M$ identity matrix, but $W_{\text {inv }} \cdot \mathbf{W}$ has only $M$ unity elements in the diagonal axis and all others are equal to zero.

If any of the eigenvalues is equal to zero, that is, if the matrix $\mathbf{R}$ is singular, these singularities can be removed simply by putting

$$
\frac{1}{w_{j}} \rightarrow 0
$$

rather than a large number in the inverse matrix $W_{\text {inv. }}$. This technique can be extended to the cases when the matrix is nearly singular, that is, when some of the eigenvalues satisfy

$$
\mathrm{w}_{\mathrm{j}}<\varepsilon \mathrm{w}_{\max }
$$

where the singularity criterion $\varepsilon$ is a preset small number and represents the desired accuracy of feedback. $\mathbf{W}_{\max }$ is the greatest of the eigenvalues. We now write $\mathbf{W}_{\mathrm{inv}}$, with the indices $i$ and $j$ in the ranges $1 \leq i \leq N$ and $1 \leq j \leq M$, as

$$
W_{i n v, i j}= \begin{cases}q_{j} \delta_{i j}, & (M \leq N) \\ q_{i} \delta_{i j}, & (M \geq N)\end{cases}
$$

where

$$
q_{j}= \begin{cases}0, & w_{j} \leq \varepsilon w_{\max } \\ \frac{1}{w_{j}}, & \text { otherwise }\end{cases}
$$

and similarly for $\mathrm{q}_{\mathrm{i}}$. For a given matrix $\mathbf{R}$, let us define $\varepsilon_{\mathrm{m}}(\mathbf{R})$ of the matrix $\mathbf{R}$ as

$$
\varepsilon_{m}(R)=\max \left\{\varepsilon \mid w_{j}>\varepsilon w_{\max } \text { for all } w_{j} \neq 0\right\} .
$$

That is, $\varepsilon_{m}$ is the largest possible value for $\varepsilon$ in order to retain all non-zero eigenvalues. Now that removal of singularities has become trivial, we will assume for simplicity that $\mathbf{R}$ is not singular in the following discussion unless noted otherwise. When $\varepsilon$ is equal to 0 , 
all the non-zero eigenvalues are kept, and we will have the most accurate feedback. However, this comes at the cost of more robust power supplies for the corrector magnets. In the other extreme case, when $\varepsilon$ is equal to $1, \mathbf{R}_{\text {inv }}$ is identically zero, and there is no feedback.

Now, from the above consideration, the pseudo-inverse of $\mathbf{R}$ defined in Eq. (2.10) satisfies

$$
\mathbf{R} \cdot \mathbf{R}_{\text {inv }} \cdot \mathbf{R}=\mathbf{R}\left(\varepsilon \leq \varepsilon_{m}\right) \quad \text { and } \quad \mathbf{R}_{\text {inv }} \cdot \mathbf{R} \cdot \mathbf{R}_{\text {inv }}=\mathbf{R}_{\text {inv }}(\text { for all } \varepsilon)
$$

In addition, with $\varepsilon \leq \varepsilon_{\mathrm{m}}$,

$$
R_{\text {inv }} \cdot R=1 \text { if } M \geq N \quad \text { and } R \cdot R_{\text {inv }}=1 \text { if } M \leq N . \quad\left(\varepsilon \leq \varepsilon_{m}\right)
$$

By removing the eigenvalues satisfying Eq. (2.12) with $\varepsilon>\varepsilon_{m}$, the inverse matrix $R_{\text {inv }}$ will be less accurate than it would otherwise be, but the vector norm of the solution can be significantly smaller. This is very desirable when certain limitations exist on the magnitude of the vector components. In our application, the solution vector is the change in the corrector strengths, which cannot be arbitrarily large because of the finite capacity of the power supplies.

Given the current orbit $\mathbf{x}_{m}$ measured by the BPMs and the desired reference orbit $\mathbf{x}_{\mathbf{r}}$, let $\Delta \mathbf{x}_{\mathbf{d}}$ be the difference orbit given by

$$
\Delta x_{d}=x_{r}-x_{m}
$$

We want to calculate back $\Delta \theta_{\mathrm{d}}$, the required changes in corrector strengths to bring the orbit to the desired reference orbit, which satisfies

$$
\mathbf{R} \cdot \Delta \boldsymbol{\theta}_{\mathrm{d}}=\Delta \mathbf{x}_{\mathrm{d}}
$$

We may categorize this linear equation according to the relative sizes of $\mathrm{M}$ and $\mathrm{N}$ as follows:

$$
\left\{\begin{array}{l}
M>N \rightarrow \text { overdetermined, no exact solutions } \\
M=N \rightarrow \text { uniquely determined, a unique solution } \\
M<N \rightarrow \text { underdetermined, many solutions }
\end{array}\right.
$$

Now, the inverse matrix $\mathbf{R}_{\text {inv }}$ obtained in Eq. (2.10) using SVD gives a solution as

$$
\Delta \boldsymbol{\theta}_{\mathrm{d}}=\mathbf{R}_{\mathrm{inv}} \cdot \Delta \mathbf{x}_{\mathrm{d}} \cdot
$$


In case $M>N$, this solution does not satisfy Eq. (2.19) exactly but minimizes the difference $\left|\mathbf{R} \cdot \Delta \boldsymbol{\theta}_{\mathrm{d}}-\Delta \mathbf{x}_{\mathrm{d}}\right|$. Consider

$$
\left|\mathbf{R} \cdot \Delta \boldsymbol{\theta}_{\mathrm{d}}-\Delta \mathbf{x}_{\mathrm{d}}\right|=\left|\mathbf{W} \cdot \Delta \boldsymbol{\theta}_{\mathrm{d}}^{\mathrm{t}}-\Delta \mathbf{x}_{\mathrm{d}}^{\mathrm{t}}\right|=\left(\sum_{\mathrm{i}=\mathrm{C}+1}^{\mathrm{M}}\left|\Delta \mathrm{x}_{\mathrm{d}, \mathrm{i}}^{\mathrm{t}}\right|^{2}\right)^{1 / 2}
$$

due to Eqs. (2.5) and (2.6). C is the number of coupled t-BPMs (or t-correctors). The index $i$ between $C+1$ and $M$ corresponds to decoupled $t-B P M s$, for which the initial difference $\Delta x_{d, i}^{t}$ cannot be changed. The coupled $t-B P M s$ will change to the reference values; therefore, Eq. (2.22) is the minimum difference and this is the best we can get.

On the other hand, when $M<N$, there are many (actually an infinite number of) solutions, and SVD picks the solution that minimizes $\left|\Delta \theta_{d}\right|$ by setting the decoupled $t-$ correctors to zero. That is,

$$
\left|\Delta \theta_{\mathrm{d}}\right|=\left|\Delta \theta_{\mathrm{d}}^{\mathrm{t}}\right|=\left(\sum_{\mathrm{j}=1}^{\mathrm{C}}\left|\Delta \theta_{\mathrm{d}, \mathrm{j}}^{\mathrm{t}}\right|^{2}\right)_{1 / 2}
$$

is the absolute minimum among all solutions, with $\Delta \theta_{\mathrm{d}, \mathrm{j}}^{\mathrm{t}}=0$ for $\mathrm{C}+1 \leq \mathrm{j} \leq \mathrm{N}$. These decoupled t-correctors do not affect orbit correction at all. So, Eq. (2.23) is the best we can get, since the overall changes in corrector strengths will be the smallest possible.

Once $\Delta \theta_{\mathrm{d}}$ as given by Eq. (2.21) is applied, the closed orbit will move to a new orbit given by

$$
\mathbf{x}_{\mathrm{m}}^{\prime}=\mathbf{x}_{\mathrm{m}}+\mathbf{R} \cdot \Delta \boldsymbol{\theta}_{\mathrm{d}}
$$

When $\varepsilon$ is larger than $\varepsilon_{m}$, this new orbit will not necessarily be equal to the reference orbit $\mathbf{x}_{\mathrm{r}}$, since $\mathbf{R} \cdot \mathbf{R}_{\mathrm{inv}}$ is not necessarily equal to 1 . However, as long as $\varepsilon$ is not changed, which keeps $\mathbf{R}_{\text {inv }}$ the same, there cannot be any further correction of the closed orbit. The new difference orbit $\Delta x_{d}^{\prime}$, from Eqs. (2.21) and (2.22), is given by

$$
\Delta x_{d}^{\prime}=x_{r}-x_{m}^{\prime}=\left(1-R \cdot R_{i n v}\right) \cdot \Delta x_{d}
$$

The new corrector strength change $\Delta \theta_{\mathrm{d}}^{\prime}$ then vanishes, since

$$
\Delta \theta_{d}^{\prime}=R_{i n v} \cdot \Delta x_{d}^{\prime}=\left(R_{\text {inv }}-R_{\text {inv }} \cdot \mathbf{R} \cdot \mathbf{R}_{\text {inv }}\right) \cdot \Delta x_{d}=0
$$

according to Eq. (2.16). In reality, due to the error in the measurement of the response matrix $\mathbf{R}$, changes in the machine condition, and external perturbations, there will remain 
some residue in the closed orbit error which still needs to be corrected. Elimination of this residue in the orbit error will be done by fast $\mathrm{AC}$ closed loop feedback with appropriate bandwidth, as is discussed in Refs. 1 and 2.

Let us then consider optimization of orbit correction by adjusting the corrector strengths such that the total vector length is minimized. In Figs. 2.1(a) and 2.1(b), three points representing the uncorrected orbit, the reference orbit, and the current orbit are shown. $\Delta \mathbf{x}_{2}$ is the difference between the reference orbit and the uncorrected orbit. The current orbit was established by applying corrector strength $\Delta \theta_{1}$ to the uncorrected orbit, which gives the current difference orbit as

$$
\Delta \mathbf{x}_{3}=\Delta \mathbf{x}_{2}-\mathbf{R} \cdot \Delta \boldsymbol{\theta}_{1}
$$

Let $\Delta \mathrm{x}_{2}^{\prime}$ and $\Delta \mathrm{x}_{3}^{\prime}$ be the corresponding residual difference orbits after correction. Then we have

$$
\Delta \mathbf{x}_{3}^{\prime}=\left(\mathbf{1}-\mathbf{R} \cdot \mathbf{R}_{\mathrm{inv}}\right) \cdot \Delta \mathrm{x}_{3}=\Delta \mathrm{x}_{2}^{\prime}-\left(\mathbf{R}-\mathbf{R} \cdot \mathbf{R}_{\mathrm{inn}} \cdot \mathbf{R}\right) \cdot \Delta \boldsymbol{\theta}_{1} .
$$

This shows that the residual difference orbit depends on the current orbit and is not unique in general. Only when $\varepsilon \leq \varepsilon_{\mathrm{m}}$, we have $\Delta \mathrm{x}_{3}^{\prime}=\Delta \mathrm{x}_{2}^{\prime}$, according to Eq. (2.16).

A similar result can be derived for the corrector strength. The incremental corrector strength $\Delta \theta_{3}$ for correction of the current orbit is given by

$$
\Delta \theta_{3}=R_{\text {inv }} \cdot \Delta \mathbf{x}_{3}=\Delta \theta_{2}-R_{\text {inv }} \cdot \mathbf{R} \cdot \Delta \theta_{1},
$$
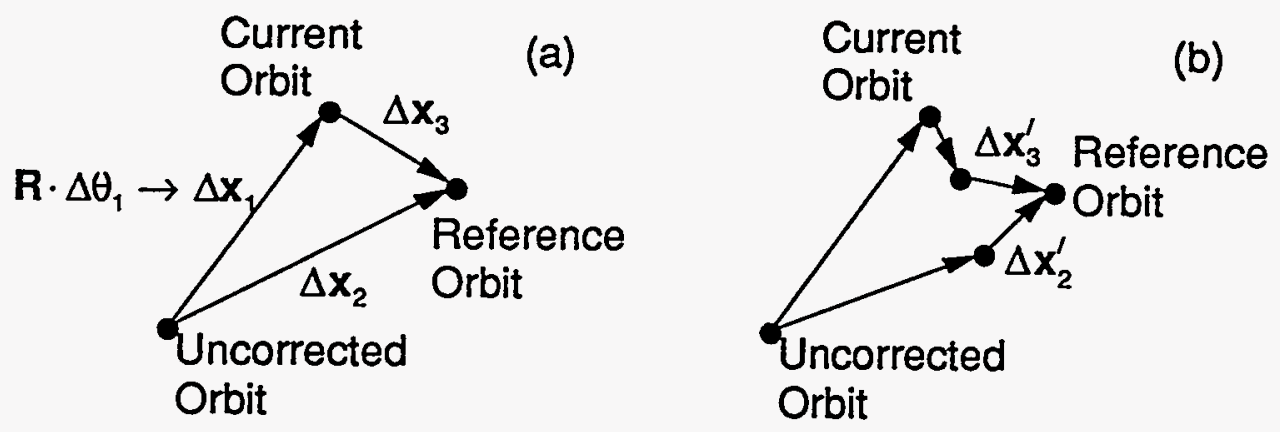

Fig. 2.1: Optimization of orbit correction. For $M \leq N$, when $\varepsilon$ is small enough such that $\mathrm{W} \cdot \mathrm{W}_{\text {inv }}=1$, we have $\Delta \mathrm{x}_{3}^{\prime}=\Delta \mathrm{x}_{2}^{\prime}$ and $\Delta \theta_{2}=\Delta \theta_{1}+\Delta \theta_{3}$. 
which gives the overall change $\Delta \theta_{31}$ as

$$
\Delta \theta_{31}=\Delta \theta_{3}+\Delta \theta_{1}=\Delta \theta_{2}+\left(1-R_{\text {inv }} \cdot R\right) \cdot \Delta \theta_{1} .
$$

When $M<N, \Delta \theta_{31}$ always has larger vector norm than $\Delta \theta_{2}$, since $\Delta \theta_{2}$ is already optimized by SVD. When $M \geq N$, we can write $\mathbf{Q}=\mathbf{1}-\mathbf{R}_{\text {inv }} \cdot \mathbf{R}$, where

$$
Q_{i j}= \begin{cases}1, & i=j \text { and } w_{j} \leq \varepsilon w_{\max } \\ 0 . & \text { otherwise }\end{cases}
$$

Therefore, only when $\varepsilon \leq \varepsilon_{\mathrm{m}}$, we have $\Delta \boldsymbol{\theta}_{31}=\Delta \theta_{2}$, and the corrector strength is unique. Generally, when $\varepsilon>\varepsilon_{\mathrm{m}}$, the corrector strength is not unique but we always have $\left|\Delta \theta_{31}\right| \geq$ $\left|\Delta \theta_{2}\right|$, since $\Delta \theta_{2} \cdot Q \cdot \Delta \theta_{1}=\Delta \theta_{2}^{t} \cdot Q \cdot \Delta \theta_{1}^{t}=0$. Besides this, when optimizing the corrector strength, it also has to be considered that the magnet current should not exceed the limit for individual correctors.

\section{Simulation of NSLS X-ray Ring}

In this section we will discuss simulation of DC global beam position feedback on the NSLS X-ray ring using the model functions $\beta$ and $\psi$, and in the next section we will present the measurement results. In Table 3.1 are shown the model $\beta$ and $\psi$ functions in the vertical direction at locations of BPMs (48) and corrector magnets (39). The nominal vertical tune of the machine is $v_{v}=6.2$. From this table, we can construct the response matrix $\mathbf{R}$ as given by Eq. (2.2) and calculate the inverse matrix $\mathbf{R}_{\mathrm{inv}}$ by using SVD. The result can be used to simulate beam position correction and estimate its efficiency in terms of the residual orbit error and the required changes in the corrector strength.

For a particle of momentum $p$, the relation between the angular deflection $\Delta \theta$ and the magnet current change $\Delta \mathrm{I}$ is obtained from

$$
\Delta \theta(\mathrm{rad})=\Delta \mathrm{I}(\mathrm{A}) \frac{0.2998}{\mathrm{p}(\mathrm{GeV} / \mathrm{c})} \frac{\mathrm{B} \ell(\mathrm{T} \cdot \mathrm{m})}{\mathrm{I}(\mathrm{A})} .
$$

For the NSLS X-ray ring, $p=2.528 \frac{\mathrm{GeV}}{\mathrm{c}}$ and $^{9}$

$$
\frac{B \ell}{\mathrm{I}}= \begin{cases}4.68 \times 10^{-4} \frac{\mathrm{T} \cdot \mathrm{m}}{\mathrm{A}}, & \text { V8 correctors } \\ 6.67 \times 10^{-4} \frac{\mathrm{T} \cdot \mathrm{m}}{\mathrm{A}}, & \text { others }\end{cases}
$$


Table 3.1: $\beta$ and $\psi$ functions (vertical) at the BPMs and correctors in the NSLS X-ray ring. The nominal tune is: $v_{v}=6.2$. (* D: disabled, $N$ : nonexistent)

\begin{tabular}{|c|c|c|c|c|c|c|}
\hline $\begin{array}{l}\text { BPM } \\
\text { Name }\end{array}$ & $\beta(m)$ & $\underset{2 \pi}{\Psi(\mathrm{rad}) /}$ & $\begin{array}{l}\text { Corrector } \\
\text { Name }\end{array}$ & $\beta(\mathrm{m})$ & $\begin{array}{c}\Psi(\mathrm{rad}) / \\
2 \pi\end{array}$ & * \\
\hline X1PUE1 & 1.553 & 0.1713 & X1V3 & 12.3800 & 0.2345 & $\bar{D}$ \\
\hline X1PUE2 & 15.9830 & 0.2498 & X1V5 & 26.4170 & 0.2559 & \\
\hline X1PUE3 & 2.2850 & 0.3679 & X1V8 & 3.4200 & 0.3343 & \\
\hline X1PUE4 & 5.3150 & 0.4660 & X1V14 & 26.4170 & 0.5191 & \\
\hline X1PUE5 & 15.9830 & 0.5252 & X1V16 & 12.3800 & 0.5405 & \\
\hline X1PUE6 & 14.0690 & 0.5504 & $\mathrm{X} 2 \mathrm{~V} 3$ & 12.3800 & 1.0095 & \\
\hline X2PUE7 & 14.0690 & 0.9995 & X2V5 & 26.4170 & 1.0309 & \\
\hline X2PUE8 & 15.9830 & 1.0248 & X2V8 & 3.4200 & 1.1093 & \\
\hline X2PUE9 & 2.2850 & 1.1429 & $\mathrm{X} 2 \mathrm{~V} 14$ & 26.4170 & 1.2940 & \\
\hline X2PUE10 & 5.3150 & 1.2410 & X2V16 & 12.3800 & 1.3155 & \\
\hline X2PUE11 & 15.9830 & 1.3002 & $\mathrm{X} 3 \mathrm{~V} 3$ & 12.3800 & 1.7845 & \\
\hline X2PUE12 & 14.0690 & 1.3254 & X3V5 & 26.4170 & 1.8059 & \\
\hline X3PUE13 & 14.0690 & 1.7745 & X3V8 & 3.4200 & 1.8843 & \\
\hline X3PUE14 & 15.9830 & 1.7998 & X3V14 & 26.4170 & 2.0691 & \\
\hline X3PUE15 & 2.2850 & 1.9179 & X3V16 & 12.3800 & 2.0905 & \\
\hline X3PUE16 & 5.3150 & 2.0160 & X4V3 & 12.3800 & 2.5595 & \\
\hline X3PUE17 & 15.9830 & 2.0752 & X4V5 & 26.4170 & 2.5809 & \\
\hline X3PUE18 & 14.0690 & 2.1004 & X4V8 & 3.4200 & 2.6593 & \\
\hline X4PUE19 & 14.0690 & 2.5495 & X4V14 & 26.4170 & 2.8440 & \\
\hline X4PUE20 & 15.9830 & 2.5748 & X4V16 & 12.3800 & 2.8655 & \\
\hline X4PUE20 & 2.2850 & 2.6929 & X4V17 & 7.3259 & 2.8853 & $\mathbf{N}$ \\
\hline X4PUE22 & 5.3150 & 2.7910 & X5V3 & 12.3800 & 3.3345 & \\
\hline X4PUE23 & 15.9830 & 2.8502 & X5V5 & 26.4170 & 3.3559 & \\
\hline X4PUE24 & 14.0690 & 2.8754 & X5V8 & 3.4200 & 3.4343 & \\
\hline X5PUE25 & 14.0690 & 3.3245 & X5V14 & 26.4170 & 3.6191 & \\
\hline X5PUE26 & 15.9830 & 3.3498 & X5V16 & 12.3800 & 3.6405 & \\
\hline X5PUE27 & 2.2850 & 3.4679 & X5V17 & 7.3259 & 3.6603 & $\mathbf{N}$ \\
\hline X5PUE28 & 5.3150 & 3.5660 & X6V3 & 12.3800 & 4.1095 & \\
\hline X5PUE29 & 15.9830 & 3.6252 & X6V5 & 26.4170 & 4.1309 & \\
\hline X5PUE30 & 14.0690 & 3.6504 & X6V8 & 3.4200 & 4.2093 & \\
\hline X6PUE31 & 14.0690 & 4.0995 & X6V14 & 26.4170 & 4.3941 & \\
\hline X6PUE32 & 15.9830 & 4.1248 & X6V16 & 12.3800 & 4.4155 & \\
\hline X6PUE33 & 2.2850 & 4.2429 & X7V3 & 12.3800 & 4.8845 & \\
\hline X6PUE34 & 5.3150 & 4.3410 & X7V5 & 26.4170 & 4.9059 & \\
\hline X6PUE35 & 15.9830 & 4.4002 & X7V8 & 3.4200 & 4.9843 & \\
\hline X6PUE36 & 14.0690 & 4.4254 & X7V14 & 26.4170 & 5.1691 & \\
\hline X7PUE37 & 14.0690 & 4.8745 & X7V16 & 12.3800 & 5.1905 & \\
\hline X7PUE38 & 15.9830 & 4.8998 & X8V3 & 12.3800 & 5.6595 & \\
\hline X7PUE39 & 2.2850 & 5.0179 & X8V5 & 26.4170 & 5.6809 & \\
\hline X7PUE40 & 5.3150 & 5.1160 & X8V8 & 3.4200 & 5.7593 & \\
\hline X7PUE41 & 15.9830 & 5.1752 & X8V14 & 26.4170 & 5.9440 & \\
\hline X7PUE42 & 14.0690 & 5.2004 & X8V16 & 12.3800 & 5.9655 & \\
\hline X8PUE43 & 14.0690 & 5.6495 & & & & \\
\hline X8PUE44 & 15.9830 & 5.6748 & & & & \\
\hline PUE45 & 2.2850 & 5.7929 & & & & \\
\hline PUE46 & 5.3150 & 5.8910 & & & & \\
\hline X8PUE47 & 15.9830 & 5.9502 & & & & \\
\hline X8PUE48 & 14.0690 & 5.9754 & & & & \\
\hline
\end{tabular}


Table 3.2: Simulation results of DC global beam position correction on the NSLS X-ray ring. $\mathbf{R}_{\text {inv }}$ was obtained for different values of $\varepsilon$ for comparison. $\varepsilon_{m}=0.00234$. The initial $\Delta \mathrm{x}_{\mathrm{rms}}$ is $207.8 \mu \mathrm{m}$.

\begin{tabular}{|l|rrrr|}
\hline$\varepsilon$ & $\Delta \mathrm{I}_{\min }(\mathrm{A})$ & $\Delta \mathrm{I}_{\max }(\mathrm{A})$ & $\Delta \mathrm{I}_{\mathrm{Ims}}(\mathrm{A})$ & $\Delta \mathrm{x}_{\mathrm{rms}}(\mu \mathrm{m})$ \\
\hline 0.001 & -4.10 & 3.39 & 1.54 & 46.8 \\
0.002 & -4.10 & 3.39 & 1.54 & 46.8 \\
0.003 & -2.64 & 1.32 & 0.85 & 72.2 \\
0.005 & -1.27 & 0.74 & 0.46 & 85.2 \\
0.01 & -1.15 & 0.27 & 0.33 & 91.6 \\
0.02 & -0.08 & 0.07 & 0.04 & 128.7 \\
0.1 & -0.08 & 0.07 & 0.04 & 128.7 \\
0.2 & -0.04 & 0.04 & 0.02 & 162.3 \\
0.3 & -0.01 & 0.01 & 0.01 & 183.1 \\
1.0 & 0.00 & 0.00 & .0 .00 & 207.8 \\
\hline
\end{tabular}

Therefore, we have from Eqs. (3.1) and (3.2)

$$
\Delta \theta(\mathrm{rad})= \begin{cases}5.55 \times 10^{-5} \Delta \mathrm{I}(\mathrm{A}), & \mathrm{V} 8 \text { correctors } \\ 7.91 \times 10^{-5} \Delta \mathrm{I}(\mathrm{A}) . & \text { others }\end{cases}
$$

The increment in the magnet current $\Delta \mathbf{I}$ can then be obtained from Eqs. (2.21) and (3.3) in terms of the difference orbit $\Delta \mathbf{x}$.

For simulation of beam position correction on the NSLS X-ray ring, a sample of uncorrected orbit with r.m.s. orbit error of $208 \mu \mathrm{m}$ relative to the reference orbit "orbit44" was taken as the initial state. The response matrix $\mathbf{R}$ was calculated from the betatron functions listed in Table 3.1. For different values of $\varepsilon$, the pseudo-inverse matrix $\mathbf{R}_{\text {inv }}$ was then calculated, which gives changes in the magnet current and resulting reduction in orbit error. The result is summarized in Table 3.2. For smaller $\varepsilon$, the r.m.s. orbit error is smaller, but the price is the larger changes in the corrector strengths.

\section{Measurement Results}

In this section, we will present the results of global vertical orbit correction experiments on the X-ray ring of NSLS. All of the 48 BPMs and 39 correctors as listed in Table 3.1 were used, except for the X1V3, X4V17, and X5V17 correctors.

The flowchart of the algorithm for DC global beam position feedback is shown in Fig. 4.1. The first step is to measure the response matrix $\mathbf{R}$. If it has already been done, 


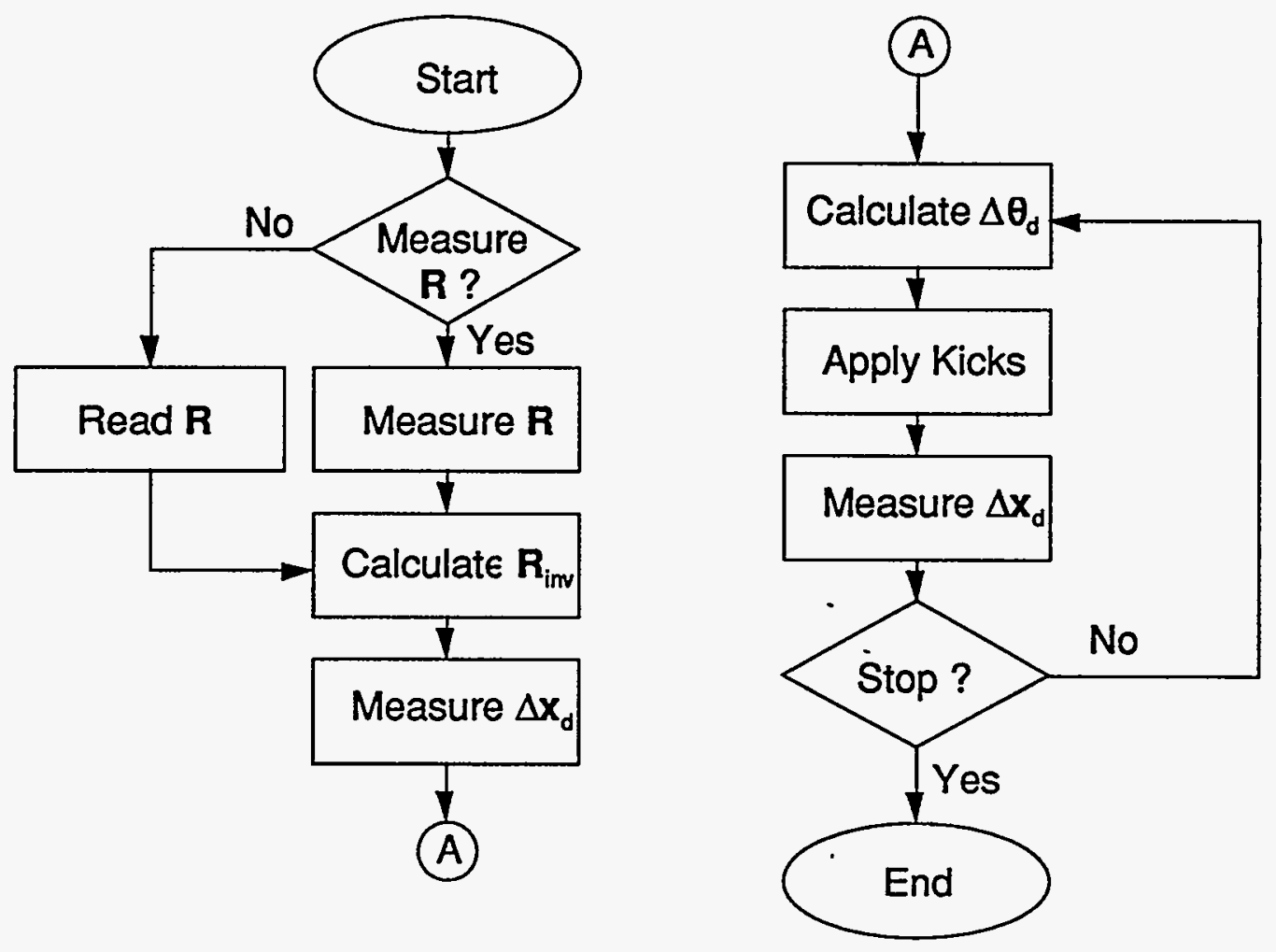

Fig. 4.1: Flowchart for DC global beam position feedback.

this step is skipped and the matrix is read from the disk. The pseudo-inverse matrix $\mathbf{R}_{\mathrm{inv}}$ is then calculated using $\varepsilon$ in Eq. (2.12) and stored in the memory. It is used to calculate the necessary kick strengths for the corrector magnets after each measurement of the difference orbit $\Delta \mathbf{x}_{\mathrm{d}}$, until it is requested by the user to stop the process.

The response matrix was measured by changing the strength of the correctors one by one and measuring the beam motion at all BPMs. From this raw response matrix, the betatron functions $\beta$ and $\psi$ were derived for the BPMs and correctors, ${ }^{10}$ which again were used to reconstruct the response matrix, thereby reducing the measurement error. For this reconstructed matrix, $\varepsilon_{\mathrm{m}}$ was 0.00182 .

Figure 4.2(a) shows the horizontal (upper) and vertical (lower) closed orbits around the ring after applying the harmonic correction to the uncorrected orbit with vertical r.m.s. orbit error of $208 \mu \mathrm{m}$. The horizontal orbit was not corrected. After harmonic correction, the vertical r.m.s. orbit error was $138 \mu \mathrm{m}$. The SVD correction was applied to this orbit, with $\varepsilon=0.002$, which further reduced the orbit error to $61 \mu \mathrm{m}$ as shown in Fig. 4.2(b). A 
(a)

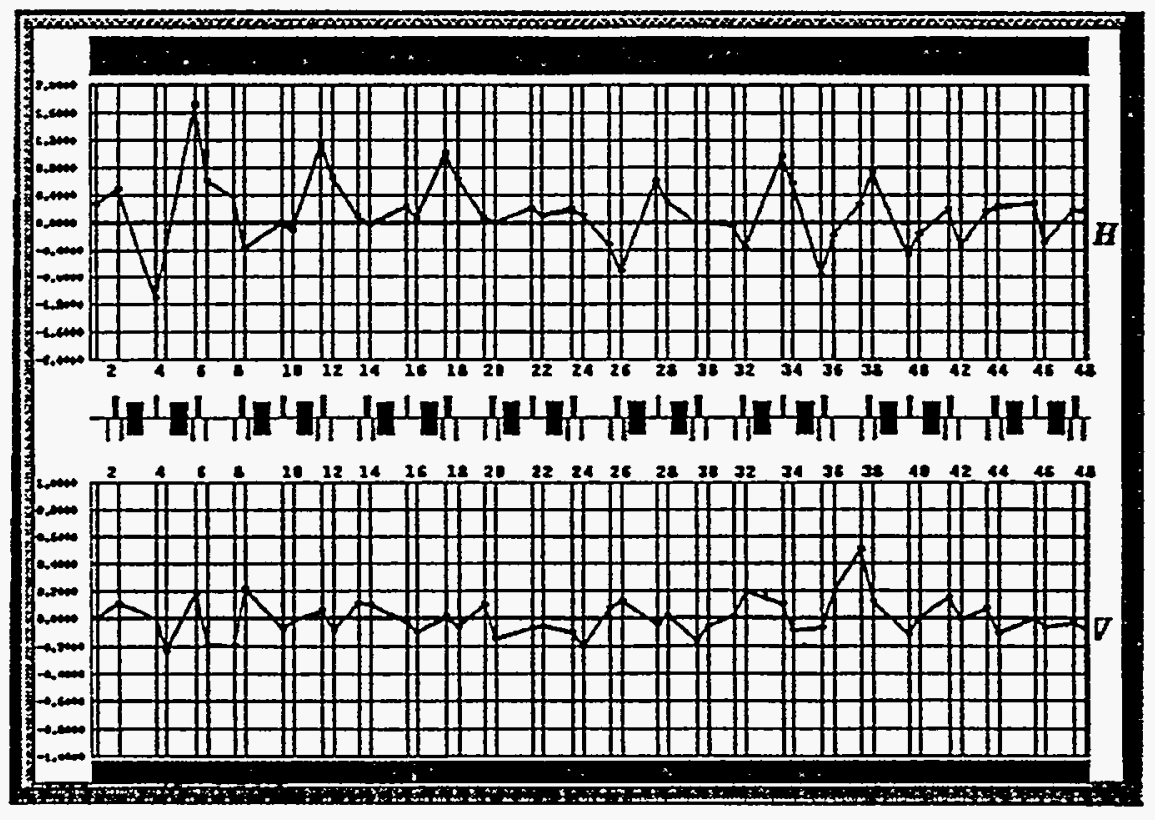

(b)

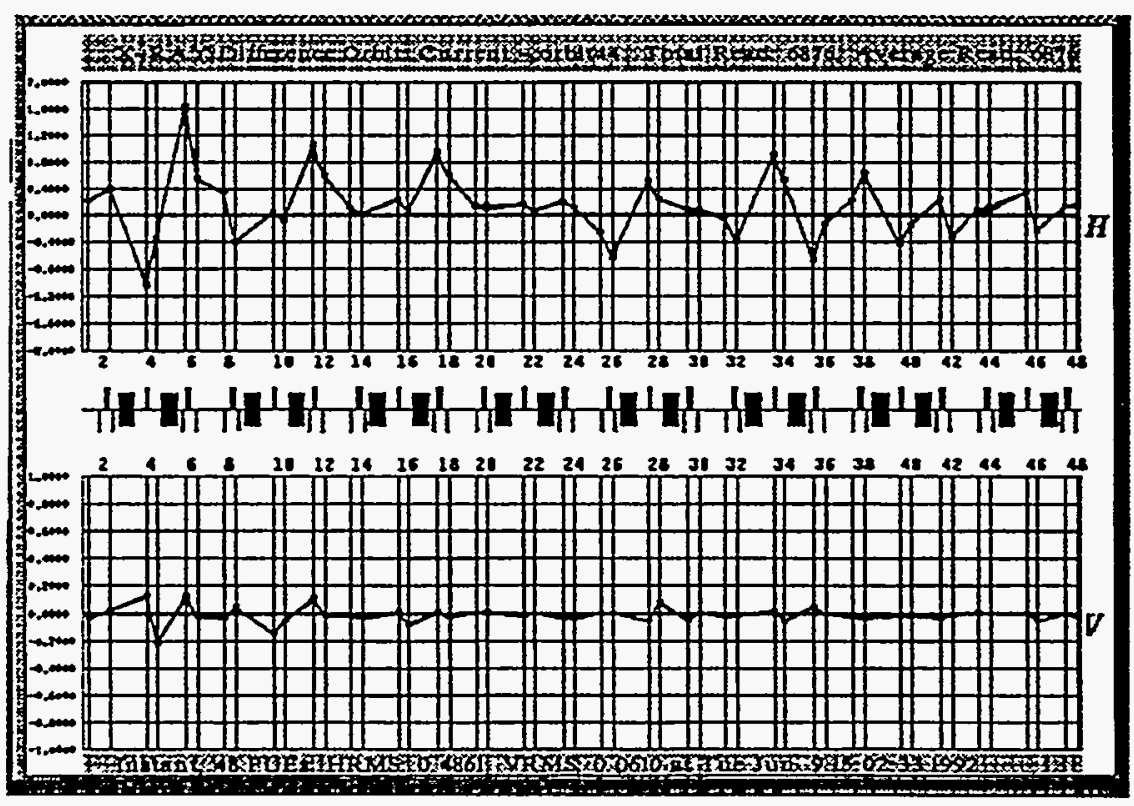

Fig. 4.2: Results of global orbit correction using (a) harmonic correction and (b) SVD correction. The uncorrected orbit had $208 \mu \mathrm{m}$ r.m.s. error relative to the reference orbit, which was reduced to $138 \mu \mathrm{m}$ by harmonic correction. SVD correction further reduced it to $61 \mu \mathrm{m}$ using $\varepsilon=0.002$. 
few corrections were necessary before the r.m.s. error settled down to this value. This is due to the difference in the machine conditions when the matrix was measured and when the orbit correction was done. While the response matrix was measured with the X25 wiggler gap closed, 11 the orbit correction was done with the gap open. This slight difference, though minor, has resulted in a less than exact correction. This was confirmed by later measurement of the response matrix with the wiggler gap open.

The corrector strength change ranged from $-4.85 \mathrm{~A}$ to $2.27 \mathrm{~A}$, with the r.m.s. value of $1.41 \mathrm{~A}$. Some of the corrector power supplies got close to, but did not reach, saturation at the maximum current of $10 \mathrm{~A}$. With $\varepsilon_{\mathrm{m}}=0.00182$, reducing $\varepsilon$ to 0.001 would trip off some of the power supplies and was not tried.

\section{Summary}

In this note, we presented the theory and application of the singular value decomposition (SVD) for DC global correction of the vertical closed orbit in the NSLS Xray ring. The method is, in principle, equivalent to inversion of the matrix, and the matrix in our case is the response matrix, which is the ratio of orbit motion per unit change in the corrector strength. Using SVD, either the residual orbit error $(M \geq N)$ or the r.m.s. corrector strength change $(M \leq N)$ is absolutely minimized. This means that given the initial difference orbit, no other correction algorithm can further reduce these. This was proven by introducing the concepts of $t$-BPMs and $t$-correctors, which are appropriate linear combinations, or transforms, of the actual BPMs and correctors.

Considering the limitation on the corrector power supplies, the important parameter is the singularity criterion $\varepsilon$ for SVD, which represents the degree of correction accuracy. For the most accurate correction, $\varepsilon$ is set to less than $\varepsilon_{\mathrm{m}}$, but this will result in large changes in the corrector strength. When this is unacceptably large due to the current limit of the power supply, $\varepsilon$ must be increased to a value less than 1 . When $\varepsilon$ is equal to 1 , there is no correction. Therefore, by adjusting $\varepsilon$, orbit corrections can be optimized in terms of the desired orbit error and the corrector strength limit.

As a result of the correction using SVD with $\varepsilon=0.002$, the r.m.s. orbit error in the vertical plane was reduced to $61 \mu \mathrm{m}$ from $138 \mu \mathrm{m}$ due to harmonic correction. The uncorrected orbit had $208 \mu \mathrm{m}$ orbit error. The corrector current change ranged from -4.85 A to $2.27 \mathrm{~A}$, with the r.m.s. of $1.41 \mathrm{~A}$.

The computer code used for this work is highly modularized so that it can be easily applied to closed orbit correction in other storage rings with proper I/O interface to the beam position monitors and corrector magnets. It also can be used for simulation and 
diagnosis of an orbit correction system if the response matrix, or alternatively the beta function, phase, and tune, is known. Such analysis for the APS storage ring is now being undertaken and will be published in the near future.

\section{Acknowledgment}

The authors would like to thank J. Safranek, I. So, Y. Tang and the operations crew of the NSLS for their hospitality and collaboration during the measurements. Thanks go to J. Galayda, A. Lumpkin and L. Teng for their continued interest and support for this work, and L. Emery is to be thanked for the useful discussions on this subject.

\section{References}

1. Y. Chung, L. Emery, and K. Kirchman, "Digital Signal Processing for Beam Position Feedback," LS Note 202, ANL, 1992.

2. Y. Chung, "Effect of Vacuum Chamber Eddy Current and Compensation by Digital Feedback," LS Note 209, ANL, 1992.

3. G. H. Golub and C. Reinsch, "Singular Value Decomposition and Least Squares Solutions," Numer. Math. 14, 403-420, 1970, and references therein. Also in J. H. Wilkinson and C. Reinsch, Linear Algebra, vol. II of Handbook for Automatic Computation, Springer-Verlag, New York, 1971.

4. G. E. Forsythe, M. A. Malcolm, and C. B. Moler, Computer Methods for Mathematical Computations, Prentice-Hall, Englewood Cliffs, N.J., 1977.

5. J. J. Dongarra, et al., LINPACK User's Guide, Chapter 11, Society for Industrial and Applied Mathematics, Philadelphia, 1979.

6. MATLAB ${ }^{\mathrm{TM}}$ User's Guide, The Mathworks, Inc., p. 3-178, 1990.

7. W. Press et al., Numerical Recipes in C, Cambridge University Press, p. 60, 1989.

8. Mathematica ${ }^{\mathrm{TM}}$, Wolfram Research, Inc., p. 454, 1988.

9. National Synchrotron Light Source Design Handbook, BNL.

10. Y. Chung and G. Decker, "New Method for Determination of $\beta$-Function and Phase," to be published as LS Note, ANL, 1992.

11. J. Safranek, private communication. 